Canadian

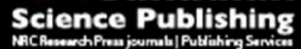

Canadian Journal of Physiology and Pharmacology Revue canadienne de physiologie et pharmacologie

\title{
Dysregulated mitogen-activated protein kinase and matrix metalloproteinase in ethanol-induced cavernosal dysfunction
}

\begin{tabular}{|r|l|}
\hline Journal: & Canadian Journal of Physiology and Pharmacology \\
\hline Manuscript ID & cjpp-2017-0082.R1 \\
\hline Manuscript Type: & Article \\
\hline Date Submitted by the Author: & $28-$ Apr-2017 \\
\hline Complete List of Authors: & $\begin{array}{l}\text { Muniz, Jaqueline Jóice; University of São Paulo (USP), Department of } \\
\text { Psychiatric Nursing and Human Sciences } \\
\text { Leite, Leticia; University of São Paulo, Department of Pharmacology } \\
\text { Lachini, Riccardo; Universidade Federal de Sao Paulo, Departamento de } \\
\text { Enfermagem Psiquiátrica e Ciências Humanas } \\
\text { Tanus-Santos, Jose; Universidade de Sao Paulo, } \\
\text { Tirapelli, Carlos; Universidade de Sao Paulo, Departamento de } \\
\text { Enfermagem Psiquiátrica e Ciências Humanas }\end{array}$ \\
\hline $\begin{array}{r}\text { Is the invited manuscript for } \\
\text { consideration in a Special } \\
\text { Issue?: }\end{array}$ & N/A \\
\hline Keyword: & $\begin{array}{l}\text { Ethanol, Endothelin-1, Mitogen-activated protein kinases, } \\
\text { Metalloproteinases, Cavernosal smooth muscle }\end{array}$ \\
\hline
\end{tabular}




\section{Dysregulated mitogen-activated protein kinase and matrix metalloproteinase in}

\section{ethanol-induced cavernosal dysfunction}

Jaqueline J. Muniz ${ }^{1}$, Letícia N. Leite ${ }^{1,2}$, Riccardo Lacchini ${ }^{1}$, José E. Tanus-Santos ${ }^{2}$, Carlos R. Tirapelli ${ }^{1}$

\footnotetext{
${ }^{1}$ Escola de Enfermagem de Ribeirão Preto, DEPCH, Universidade de São Paulo (USP), Ribeirão Preto, SP, Brazil.

${ }^{2}$ Departamento de Farmacologia, Faculdade de Medicina de Ribeirão Preto, USP, Ribeirão Preto, SP, Brazil.
}
*Corresponding author: Escola de Enfermagem de Ribeirão Preto - USP, Laboratório de Farmacologia, Avenida Bandeirantes 3900, CEP 14040-902, Ribeirão Preto, SP, Brazil, e-mail address: jaquelinejoice@yahoo.com.br, Phone: +55-16-3315-0532, FAX number: +55-16-3315-0518.




\begin{abstract}
We evaluated the effects of ethanol consumption on the mitogen-activated protein kinases (MAPK) and metalloproteinases (MMP) pathways in the rat cavernosal smooth muscle (CSM). Male Wistar rats were treated with ethanol (20\% vol./vol.) for six weeks. Quantitative real-time polymerase chain reaction experiments showed that ethanol consumption did not alter mRNA levels of p38MAPK, SAPK/JNK, ERK1/2, MMP-2 or MMP-9 in the rat CSM. Western immunoblotting experiments revealed decreased protein expression of p38MAPK and phosphorylation of SAPK/JNK in the CSM from ethanol-treated rats. Additionally, ethanol consumption decreased the expression of MMP-2. Functional assays showed that SP600125, an inhibitor of SAPK/JNK, prevented the increase in endothelin (ET)-1-induced contraction in the CSM from ethanol-treated rats. Treatment with ethanol decreased MMP-2 activity, but did not change net MMP activity in the rat CSM. Ethanol consumption increased the circulating levels of MMP-2, MMP-9 and TIMP-2 as well as the ratio MMP-9/TIMP-1. The major finding of our study is that ethanol consumption down-regulates both MAPK and MMP pathways in the rat CSM, while it increases the circulating levels of MMP-9. Additionally, we found that SAPK/JNK plays a role in ethanol-induced increase on ET1 contraction in the isolated rat CSM.
\end{abstract}

\title{
Keywords
}

Ethanol; Endothelin-1; Mitogen-activated protein kinases; Metalloproteinases; Cavernosal smooth muscle 


\section{Introduction}

Chronic ethanol consumption is one of the main causes of erectile dysfunction (ED) of vascular origin (Peugh and Belenko 2001). Several studies have established a positive relationship between ED and ethanol consumption (Grinshpoon et al. 2007; Ponizovsky 2008), but the mechanisms underlying ethanol-induced ED are not fully understood. Recently, we provided evidence that chronic ethanol consumption decreased intracavernosal pressure in rats (Muniz et al. 2015a). This response was accompanied by increased levels of circulating endothelin (ET)-1, increased generation of reactive oxygen species (ROS) and increased expression of ET-1 and $\mathrm{ET}_{\mathrm{A}}$ receptors in the rat cavernosal smooth muscle (CSM) (Muniz et al. 2015a; Leite et al. 2013). Additionally, previous findings from our laboratory showed that ethanol consumption decreased the relaxation induced by acetylcholine and increased the contractility of the rat CSM to both ET-1 and electrical field stimulation (Muniz et al. 2015a; Lizarte et al. 2009). Therefore, changes in CSM reactivity could play a role in ethanol-induced ED.

Mitogen-activated protein kinases (MAPK) are a family of serine/threonine kinases and the following members of the MAPK family are the best characterized: extracellular signal-regulated kinases (ERK1/2), p38MAPK, and stress-activated protein kinase/c-Jun N-terminal kinases (SAPK/JNK) (Pearson et al. 2001). MAPK are redoxsensitive proteins, which are described to play a role in distinctive cellular events such as apoptosis, cell differentiation, contraction and matrix remodeling (Pearson et al. 2001; Kwon et al. 2003). Activation of the MAPK pathway is associated with hypertension- and aging-induced ED (Carneiro et al. 2008; Castela et al. 2011). Modulation of the MAPK signaling pathway by ethanol is cell-specific and is related to the dose of ethanol and the period of treatment with ethanol (Aroor and Shukla 2004). In this sense, ethanol is described to alter the expression and/or phosphorylation of 
MAPK, leading to the activation or inhibition of these kinases (Aroor and Shukla 2004; Sachinidis et al. 1999; Passaglia et al. 2015; Marchi et al. 2016). However, the effect of chronic ethanol consumption on the MAPK pathway in the CSM and the contribution of this response to CSM contraction are unknown.

Metalloproteinases (MMP) are a family of zinc-dependent proteinases that degrade proteins of the extracellular matrix (Visse and Nagase 2003). MMP-2 and -9 are the main MMP involved in tissue/vascular remodeling and for this reason are associated with several cardiovascular diseases (Belo et al. 2015). Physiologically, MMP are inhibited by the tissue inhibitors of MMP (TIMP). TIMP-1 and -2 inhibit MMP-9 and MMP-2, respectively, and disruption of the balance between MMP and their inhibition by TIMP may result in tissue remodeling (Nagase and Brew 2003; Brew and Nagase 2010). Long-term ethanol consumption increases MMP-2 activity in the kidney and aorta (Partridge et al. 1999; Tirapelli et al. 2012b), and it is also associated with increased levels of circulating MMP-9 (Sillanaukee et al. 2002). MMP are expressed in the CSM (Sirad et al. 201), but the effect of ethanol consumption in MMP activity or expression in this tissue remains elusive.

While there are reports describing that ethanol modulate both MAPK and MMP pathways, the effect of ethanol on these redox-sensitive pathways in the CSM remains elusive. Here, we investigated the effects of ethanol consumption on components of the MAPK pathway and the contribution of MAPK to ethanol-induced hyper-contractility of the CSM to ET-1. The effects of ethanol consumption on the MMP pathway in the CSM and plasma were also investigated. 


\section{Materials and Methods}

\section{Experimental design}

Male Wistar rats were purchased from the animal facility of the Campus of Ribeirao Preto, University of São Paulo, Ribeirão Preto, Brazil. The rats, initially weighing 260-300 g (50-70 days old), were randomly divided into two groups: control and ethanol. Rats from the control group had access to filtered water ad libitum, while animals from the ethanol group had free access to ethanol $20 \%$ (vol./vol.) as described previously (Muniz et al. 2015a; Leite et al. 2013). Ethanol-treated rats were submitted to a three-week period of adaptation in which increasing concentrations of ethanol were provided as follows: 5, 10 and $20 \%$ of ethanol in the first, second and third week, respectively. After adaptation, rats were treated for six weeks with ethanol $20 \%$. The animals were cared for in accordance with the Guide to the Care and Use of Experimental Animals from Canadian Council on Animal Care (CCAC). All protocols were approved by the Animal Ethics Committee of the University of São Paulo Campus of Ribeirão Preto (\#12.1.317.53.9).

\section{Quantitative real-time polymerase chain reaction}

Quantitative analysis of the genes of interest was performed in the rat CSM as previously described (Leite et al. 2013). The following genes were analyzed: MMP-2 (Rn0153817_m1), MMP-9 (Rn00579162_m1), p38MAPK (Rn00578842_m1), SAPK/JNK (Rn00588007_m1) and ERK1/2 (Rn00569058_m1). Reactions were carried out in duplicate and analyzed with a StepOne Plus Real Time PCR machine (Applied Biosystems, Carlsbad, CA, USA). A relative quantification was performed using the $2^{-\Delta \Delta \mathrm{Ct}}$ method. The GAPDH gene ( $\left.\mathrm{Rn} 01775763\right)$ was used as the housekeeping gene and a group of eight control animals as calibrators. 


\section{Western immunoblotting}

Preparation of the samples and separation of the proteins were performed as previously described (Muniz et al. 2015a; Leite et al. 2013). Nitrocellulose membranes were incubated overnight at $4{ }^{\circ} \mathrm{C}$ with one of the following primary antibodies: antip38MAPK [9212S, diluted 1:1000, Cell Signaling, Danvers, MA, USA], anti-phosphop38MAPK $\left(\mathrm{Thr}^{180} / \mathrm{Tyr}^{182}\right.$ ) [9211S, diluted 1:1000, Cell Signaling], anti- SAPK/JNK [9252S, diluted 1:1000, Cell Signaling], anti-phospho-SAPK/JNK $\left(\mathrm{Thr}^{183} / \mathrm{Tyr}^{185}\right.$ ) [4668S, diluted 1:1000, Cell Signaling], anti-ERK1/2 [9102, diluted 1:1000, Cell Signaling], anti-phospho-ERK1/2 $\left(\mathrm{Thr}^{202} / \mathrm{Tyr}^{204}\right)$ [9101, diluted 1:1000, Cell Signaling], anti-MMP-9 [110186, diluted 1:1000, Abcam, Cambridge, UK], anti-MMP-2 [38898, diluted 1:1000, Abcam, Cambridge, UK], anti-TIMP-1 [AB770, diluted 1:250, Merck Millipore, Darmstadt, Germany] or anti-TIMP-2 [MAB13446, diluted 1:500, Merck Millipore]. Membranes where washed out and then incubated with secondary antibodies for $90 \mathrm{~min}$ at room temperature. A ChemiDoc ${ }^{\mathrm{TM}}$ XRS (Bio-Rad, Hercules, CA, USA) was used to visualize the signals, which were quantified densitometrically. Beta-actin [sc-47778, diluted 1:2500, Santa Cruz Biotechnology, Dallas, TX, USA] was used as an internal control.

\section{Gelatin zymography for detection of MMP-9 and MMP-2}

Gelatin zymography was performed for detection of MMP-9 and MMP-2 in plasma and CSM samples as previously described (Sirad et al. 2011). Gelatinolytic activity was determined as unstained bands against a blue stained background. The enzymatic activity was determined by densitometry using the program ImageJ (National Institutes of Health, USA). MMP-2 and MMP-9 were identified as 72 and $92 \mathrm{kDa}$ bands, respectively, by direct comparison with low molecular weight protein marker for 
Sodium dodecyl sulfate-Polyacrylamide gel electrophoresis (SDS-PAGE). Results are expressed as arbitrary units.

\section{Determination of MMP and TIMP levels in plasma}

Plasma levels of MMP-9, MMP-2, TIMP-1 and TIMP-2 were measured by enzyme-linked immunosorbent assay (ELISA) using the following commercially available kits: rat MMP-9 (E90553Ra, USCNLIFE, China), rat MMP-2 (E90100Ra, USCNLIFE, China), rat TIMP-1-Tissue Inhibitors of Metalloproteinase 1 (MBS2502910, MyBiosource, San Diego, CA, USA) and rat TIMP-2-Tissue Inhibitors of Metalloproteinase 2 (MBS2504314, MyBiosource, San Diego, CA, USA). Results are expressed as pg/ml of plasma for MMP-9, MMP-2 and TIMP-1 and as ng/ml of plasma for TIMP-2.

\section{Determination of gelatinolytic activity in the rat CSM}

Gelatinolytic activity was determined fluorometrically using dye-quenched (DQ)-gelatin as a substrate. The rat CSM was vertically embedded in Tissue-tek ${ }^{\circledR}$ and sectioned transversely (10- $\mu$ m-thick slices). The slices were incubated with DQ-gelatin (E12055, Molecular Probes, OR, USA) at a concentration of $1 \mathrm{mg} / \mathrm{ml}$ in Tris- $\mathrm{CaCl}_{2}$ buffer (Tris $50 \mathrm{mM}, \mathrm{CaCl}_{2} 10 \mathrm{mM}$ and $\mathrm{ZnCl}_{2} 1 \mathrm{mM}$ ) for $60 \mathrm{~min}$, and then washed 3 times with cold phosphate buffered saline (PBS, pH 7.4). Sections were examined by fluorescence microscopy (Axio Vert A1, Carl Zeiss, Jena, Germany). Wavelengths of excitation and emission were 480 and $510 \mathrm{~nm}$, respectively. The images were captured at $\times 400$ and analyzed using the program ImageJ (National Institutes of Health, USA) (Rizzi et al. 2014). Results are expressed as arbitrary units.

\section{Functional studies}

The rat CSM was isolated and mounted in 5-ml organ chambers as previously described (Muniz et al. 2015a; Leite et al. 2013). The strips were stretched until they 
reached a resting tension of $3 \mathrm{mN}$ and allowed to equilibrate for $60 \mathrm{~min}$. After equilibration, each strip was stimulated with $\mathrm{KCl}(120 \mathrm{mM})$. The strips were then sequentially washed and allowed to relax to baseline. Cumulative concentrationresponse curves for ET-1 $(0.1 \mathrm{nM}-1 \mu \mathrm{M})$ were performed in the rat CSM by a stepwise increase in the concentration of the peptide. Since ethanol treatment did not alter the contraction induced by $\mathrm{KCl} 120 \mathrm{mM}$ (Muniz et al. 2015a; Leite et al. 2013), the contraction induced by ET-1 is expressed as percentage of the contraction induced by $\mathrm{KCl} 120 \mathrm{mM}$. ET-1-induced contraction was evaluated in the absence (control) or after incubation for 30 min with one of the following drugs: SB203580 (p38MAPK inhibitor, $100 \mu \mathrm{M}$; Sigma-Aldrich, St. Louis, MO, USA), SP600125 (SAPK/JNK inhibitor, $100 \mu \mathrm{M}$; Sigma-Aldrich) or PD98059 (ERK1/2 inhibitor, $100 \mu \mathrm{M}$; SigmaAldrich). The concentrations of the inhibitors were based on previous studies (Kwon et al. 2003; Lee et al. 2007). The concentration-response curves for ET-1were fitted using the nonlinear interactive fitting program GraphPad Prism® ${ }^{\circledR} .01$ (GraphPad Software Inc., San Diego, CA, USA). Agonist potencies and maximal responses are expressed as $\mathrm{pD}_{2}$ (negative logarithm of the molar concentration of agonist producing $50 \%$ of the maximal response) and $\mathrm{E}_{\max }$ (maximum effect elicited by the agonist), respectively.

\section{Statistical analysis}

Statistical analysis was performed using the program GraphPad Prism® 5.01 (GraphPad Software Inc., San Diego, CA, USA). Data are presented as the means \pm standard error of the mean (S.E.M.). Results were compared using Student's $t$ test or one-way analysis of variance (ANOVA) followed by Bonferroni's comparison test. Results of statistical tests with $\mathrm{P}<0.05$ were considered as significant. 


\section{Results}

Effects of chronic ethanol consumption on mRNA levels and protein expression of $M A P K$ and MMP in the rat CSM

Chronic ethanol consumption did not alter mRNA levels of p38MAPK, SAPK/JNK, ERK1/2, MMP-9 or MMP-2 (Table 1). Decreased expression of p38MAPK was detected in the CSM from ethanol-treated rats, when compared to control ones (Fig. 1A). On the other hand, treatment with ethanol did not alter the expression of SAPK/JNK or ERK1/2 (Fig. 1C and E). CSM from ethanol-treated rats displayed decreased SAPK/JNK phosphorylation, when compared with control ones (Fig. 1D). Treatment with ethanol did not affect p38MAPK or ERK1/2 phosphorylation in the rat CSM (Fig. 1B and F).

Treatment with ethanol did not alter MMP-9 expression in the rat CSM (Fig. 2A). Protein expression of MMP-2 was decreased in the CSM from ethanol-treated rats, when compared with control ones (Fig. 2B). On the other hand, the protein expression of TIMP-1 and TIMP-2 was not affected by ethanol consumption (Fig. 2C and D). Treatment with ethanol did not affect MMP-9/TIMP-1 ratio, but increased MMP2/TIMP-2 ratio in the rat CSM (Fig. 2E and F).

\section{Effects of chronic ethanol consumption in the rat CSM reactivity to ET-1}

Ethanol consumption increased the contractile response to ET-1 in the rat CSM (Table 2). SP600125 was used to evaluate the role of SAPK/JNK in ethanol-induced hyper-contractility of the rat CSM to ET-1. SP600125 reduced the contractile response to ET-1 in the CSM from ethanol-treated rats (Table 2). In order to evaluate the involvement of p38MAPK and ERK1/2 in the hyper-contractility to ET-1, the rat CSM was incubated with SB203580 and PD98059, respectively. Our results show that neither SB203580 nor PD98059 affected ET-1-induced contraction in the CSM from ethanol- 
treated rats (Table 2). No differences in $\mathrm{pD}_{2}$ values were found among experimental groups (Table 2).

\section{Effects of chronic ethanol consumption in MMP activity in the rat CSM}

Treatment with ethanol did not alter the net MMP activity in the rat CSM (Fig. 3A and B). On the other hand, chronic ethanol consumption decreased the activity of MMP-2 in the rat CSM (Fig. 3C and D).

\section{Effects of chronic ethanol consumption in MMP levels and activity in the rat plasma}

Treatment with ethanol increased plasma levels of both MMP-9 and MMP-2 (Fig. 4A and B). No differences in plasma TIMP-1 levels were observed after treatment with ethanol (Fig. 4C). However, increased plasma levels of TIMP-2 were detected after treatment with ethanol (Fig. 4D). Treatment with ethanol increased MMP-9/TIMP-1 ratio, whereas no change in MMP-2/TIMP-2 ratio was detected in the plasma from ethanol-treated rats (Fig. 4E and F). Finally, ethanol consumption did not alter the activity of MMP-9 or MMP-2 in the rat plasma (Fig. 5A and B).

\section{Discussion}

Our findings show that ethanol consumption did not affect mRNA levels of p38MAPK, but decreased its expression in the rat CSM, suggesting that ethanol affected p38MAPK expression at a post-transcriptional level. This result is in accordance with previous findings in the vasculature where ethanol consumption was found to modulate protein expression at a post-transcriptional level (Tirapelli et al. 2006a; Tirapelli et al. 2008b). The exact cellular/functional meaning of the reduction of p38MAPK expression induced by ethanol in the rat CSM is not well understood. In fact, down-regulation of the MAPK pathway is a compensatory mechanism in some conditions such as hypertension (Kim et al. 2004). Importantly, we have described that 
ethanol consumption increased both blood pressure and CSM reactivity, while it decreased the intracavernosal pressure (Muniz et al. 2015a; Leite et al. 2013; Lizarte et al. 2009; Passaglia et al. 2015). Thus, the reduced p38MAPK expression here described may be an intrinsic adaptive response to ethanol-induced increase on CSM reactivity and blood pressure.

The present findings show that CSM from ethanol-treated rats displayed decreased SAPK/JNK phosphorylation, while phosphorylation of both ERK1/2 and p38MAPK was not affected by ethanol consumption. MAPK are activated by phosphorylation cascades being the kinase immediately upstream of the MAPK a member of the MEK (or MKK) family. Each member of the MEK family phosphorylates only a few or one member of the MAPK family (Pearson et al. 2001). MEK4/7 is responsible for the phosphorylation of SAPK/JNK, while MEK1/2 and MEK3/6 phosphorylates ERK1/2 and p38MAPK, respectively (Pearson et al. 2001). Thus, the distinctive effect of ethanol on MAPK phosphorylation in the rat CSM may be the result of the complex signaling networks that underlie MAPK activation, which requires MAPK phosphorylation by different members of the MEK family.

MAPK regulate the $\mathrm{Ca}^{2+}$-independent contraction induced by ET-1 in distinctive tissues (Pollock et al. 1995). Our functional results showed that ethanol consumption increased ET-1-induced contraction in the rat CSM, which is in line with previous reports (Muniz et al. 2015a; Leite et al. 2013). SP600125 reversed the increased reactivity of the CSM to ET-1, suggesting a role for SAPK/JNK in such response. Although SP600125 reversed the hyper-reactivity to ET-1 induced by ethanol, treatment with ethanol paradoxically decreased SAPK/JNK phosphorylation. However, it is important to note that our results show the phosphorylation of SAPK/JNK in basal conditions and not the phosphorylation triggered by ET-1. 
Ethanol consumption is described to modulate the expression and activity of MMP in distinctive tissues (Partridge et al. 1999; Sillanaukee et al. 2002). The present findings first demonstrated that ethanol consumption did not change mRNA levels of MMP-2 or MMP-9, but it decreased the expression and activity of MMP-2 in the rat CSM. These results suggested that ethanol consumption decreased MMP-2 expression at a post-transcriptional level. MMP are regulated by TIMP (Nagase and Brew 2003), and disruption of the balance between MMP and their inhibition by TIMP may result in tissue remodeling (Brew and Nagase 2010). For this reason MMP/TIMP ratio is considered to be a better parameter to evaluate MMP actions than MMP expression/dosage (Muniz et al. 2012b). We found that ethanol consumption did not change TIMP-1 and TIMP-2 expression in the rat CSM. Thus, while MMP-9/TIMP-1 ratio was not affected by ethanol a decreased MMP-2/TIMP-2 ratio was detected in the CSM from ethanol-treated rats.

The net MMP activity in the rat CSM was not affected by ethanol, which is in accordance with previous findings in the mesenteric arterial bed (Rocha et al. 2012). However, treatment with ethanol decreased MMP-2 activity in the rat CSM. This finding contrasts previous results describing that ethanol did not change MMP-2 activity in the rat liver and mesenteric arterial bed (Rocha et al. 2012; Tirapelli et al. 2011a), and increased MMP-2 activity in the rat kidney (Sillanaukee et al. 2002). Based on such observations, we concluded that the effects of ethanol on MMP activity are tissuespecific. Another point that could explain these discrepancies is the period of treatment with ethanol. For example, Partridge et al. (1999) observed an increase on MMP-2 activity in aortas of rats treated with ethanol $35 \%$ for 72 weeks, while we have previously found that treatment for 6 weeks with ethanol $20 \%$ did not alter MMP-2 activity in the rat aorta (Hipólito et al. 2011). 
Circulating MMP are used as biomarkers in a variety of pathological conditions, including ED (Muniz et al. 2012b). Our findings show that ethanol consumption increased MMP-9 levels without affecting its activity. This result is in line with a previous study showing increased circulating levels of MMP-9 in alcoholic individuals (Sillanaukee et al. 2002). Moreover, ethanol did not change plasma TIMP-1 levels, which is a physiological inhibitor of MMP-9. Treatment with ethanol also increased plasma MMP-2 levels. However, we must be aware of the fact that increased TIMP-2 levels were also detected after ethanol treatment. In this sense, despite the increase in MMP-2 levels, no difference on MMP-2/TIMP-2 ratio was observed.

Using this model of ethanol treatment we have previously found blood ethanol levels in the range of 35-40 mM (Muniz et al. 2015a; Leite et al. 2013). Thus, the present findings are of physiological significance since similar blood ethanol concentrations are described in alcoholic individuals (Urso et al. 1981).

The major finding of our study is that ethanol consumption down-regulates both MAPK and MMP pathways in the rat CSM, while it increases the circulating levels of MMP-9. Additionally, we found that SAPK/JNK plays a role in ethanol-induced increase on ET-1 contraction in the isolated rat CSM.

\section{Funding}

This study was supported by Fundação de Amparo à Pesquisa do Estado de São Paulo, Brazil (grants \#2011/12911-2 and \#2012/04144-4) and Conselho Nacional de Desenvolvimento Científico e Tecnológico, Brazil (grant \#470556/2010-2).

\section{Conflict of interest}

The authors declare no conflict of interest. 


\section{References}

Aroor, A.R. and Shukla, S.D. 2004. MAP kinase signaling in diverse effects of ethanol. Life Sci. 74(19): 2339-2364.

Belo, V.A., Guimarães, D.A., and Castro, M.M. 2015. Matrix Metalloproteinase 2 as a Potential Mediator of Vascular Smooth Muscle Cell Migration and Chronic Vascular Remodeling in Hypertension. J. Vasc. Res. 52(4): 221-231.

Brew, K. and Nagase, H. 2010. The tissue inhibitors of metalloproteinases (TIMPs): an ancient family with structural and functional diversity. Biochim. Biophys. Acta. 1803(1): 55-71.

Carneiro, F.S., Nunes, K.P., and Giachini, F.R., et al. 2008. Activation of the ET-1/ETA pathway contributes to erectile dysfunction associated with mineralocorticoid hypertension. J. Sex. Med. 5(12): 2793-2807.

Castela, A., Soares, R., and Rocha, F., et al. 2011 Erectile tissue molecular alterations with aging: differential activation of the p42/44 MAP Kinase pathway. Age (Dordr). 33(2): 119-130.

Grinshpoon A, Margolis A, Weizman A, et al. 2007. Sildenafil citrate in the treatment of sexual dysfunction and its effect on quality of life in alcohol dependent men: preliminary findings. Alcohol Alcohol. 42(4): 340-346.

Hipólito, U.V., Rocha, J.T., and Martins-Oliveira, A., et al. 2011. Chronic ethanol consumption reduces adrenomedullin-induced relaxation in the isolated rat aorta. Alcohol. 45(8): 805-814.

Kim, B., Kim, J., and Bae, Y.M., et al. 2004. p38 mitogen-activated protein kinase contributes to the diminished aortic contraction by endothelin-1 in DOCA-salt hypertensive rats. Hypertension. 43(5): 1086-1091. 
Kwon, S., Lee, W.J., and Fang, L.H., et al 2003. Mitogen-activated protein kinases partially regulate endothelin-1-induced contractions through a myosin light chain phosphorylation-independent pathway. J. Vet. Med. Sci. 65(2): 225-230.

Lee, H.M., Won, K.J., and Kim, J., et al. 2007. Endothelin-1 induces contraction via a Syk-mediated p38 mitogen-activated protein kinase pathway in rat aortic smooth muscle. J. Pharmacol. Sci. 103(4): 427-433.

Leite, L.N., Lacchini, R., and Carnio, E.C., et al. 2013. Ethanol consumption increases endothelin-1 expression and reactivity in the rat cavernosal smooth muscle. Alcohol Alcohol. 48(6): 657-666.

Lizarte, F.S., Claudino, M.A., and Tirapelli, C.R., et al. 2009. Chronic ethanol consumption induces cavernosal smooth muscle dysfunction in rats. Urology. 74 (6):1250-1256.

Marchi, K.C., Ceron, C.S., and Muniz, J.J., et al. 2016. NADPH Oxidase Plays a Role on Ethanol-Induced Hypertension and Reactive Oxygen Species Generation in the Vasculature. Alcohol Alcohol. 51(5): 522-534.

Muniz, J.J., Lacchini, R., and Belo, V.A., et al. 2012b. Circulating matrix metalloproteinases and their endogenous inhibitors in patients with erectile dysfunction. Int. J. Impot. Res. 24(1): 38-43.

Muniz, J.J., Leite, L.N., and De Martinis, B.S., et al. 2015a. Chronic ethanol consumption induces erectile dysfunction: Role of oxidative stress. Life Sci. 141: 44-53.

Nagase, H. and Brew, K. 2003. Designing TIMP (tissue inhibitor of metalloproteinases) variants that are selective metalloproteinase inhibitors. Biochem. Soc. Symp. 70: 201-212. 
Partridge, C.R., Sampson, H.W. and Forough, R. 1999. Long-term alcohol consumption increases matrix metalloproteinase-2 activity in rat aorta. Life Sci. 65(13): 13951402.

Passaglia, P., Ceron, C.S., and Mecawi, A.S., et al. 2015. Angiotensin type 1 receptor mediates chronic ethanol consumption-induced hypertension and vascular oxidative stress. Vascul. Pharmacol. 74: 49-59.

Pearson, G., Robinson, F., and Beers Gibson, T., et al. 2001. Mitogen-activated protein kinase pathways: regulation and physiological functions. Endocr. Rev. 22: 153-183.

Peugh, J. and Belenko, S. 2001. Alcohol, drugs and sexual function: a review. J Psychoactive Drugs. 33(3): 223-232.

Pollock, D.M., Keith, T.L., and Highsmith, R.F. 1995. Endothelin receptors and calcium signaling. FASEB J. 9: 1196-1204.

Ponizovsky, A.M. 2008. Clinical and psychosocial factors associated with quality of life in alcohol-dependent men with erectile dysfunction. J. Sex. Med. 5(10): 2347-2358.

Rizzi, E., Guimaraes, D.A., and Ceron, C.S., et al. 2014. $\beta 1$-Adrenergic blockers exert antioxidant effects, reduce matrix metalloproteinase activity, and improve renovascular hypertension-induced cardiac hypertrophy. Free Radic. Biol. Med. 73: 308-317.

Rocha, J.T., Hipólito, U.V., and Martins-Oliveira, A., et al. 2012. Ethanol consumption alters the expression and reactivity of adrenomedullin in the rat mesenteric arterial bed. Alcohol Alcohol. 47(1): 9-17.

Sachinidis, A., Gouni-Berthold, I., and Seul, C., et al. 1999. Early intracellular signalling pathway of ethanol in vascular smooth muscle cells. Br. J. Pharmacol. 128(8): 1761-1771. 
Sillanaukee, P., Kalela, A., and Seppä, K., et al. 2002. Matrix metalloproteinase-9 is elevated in serum of alcohol abusers. Eur. J. Clin. Invest. 32(4): 225-229.

Sirad, F., Hlaing, S., and Kovanecz, I., et al. 2011. Sildenafil promotes smooth muscle preservation and ameliorates fibrosis through modulation of extracellular matrix and tissue growth factor gene expression after bilateral cavernosal nerve resection in the rat. J. Sex. Med. 8(4): 1048-1060.

Tirapelli, C.R., Casolari, D.A., and Montezano, A.C., et al. 2006a. Ethanol consumption enhances endothelin-1-induced contraction in the isolated rat carotid. J. Pharmacol. Exp. Ther. 318(2): 819-827.

Tirapelli, C.R., Leone, A.F., and Yogi, A., et al. 2008b. Ethanol consumption increases blood pressure and alters the responsiveness of the mesenteric vasculature in rats. J. Pharm. Pharmacol. 60(3): 331-341.

Tirapelli, L.F., Batalhão, M.E., and Jacob-Ferreira, A.L., et al. 2011a. Chronic ethanol consumption induces histopathological changes and increases nitric oxide generation in the rat liver. Tissue Cell. 43(6): 384-391.

Tirapelli, L.F., Martins-Oliveira, A., and Batalhão, M.E., et al. 2102b. Ethanol consumption increases the expression of endothelial nitric oxide synthase, inducible nitric oxide synthase and metalloproteinases in the rat kidney. J. Pharm. Pharmacol. 64(1): $68-76$.

Urso, T., Gavaler, J.S., and Van Thiel, D.H. 1981. Blood ethanol levels in sober alcohol users seen in an emergency room. Life Sci. 28: 1053-1056.

Visse, R. and Nagase, H. 2003. Matrix metalloproteinases and tissue inhibitors of metalloproteinases: structure, function, and biochemistry. Circ. Res. 92(8): 827839. 
Table 1 mRNA expression of p38MAPK, SAPK/JNK, ERK1/2, MMP-9 and MMP-2 in the CSM from control and ethanol-treated rats.

\begin{tabular}{ccc}
\hline Fold $2^{-\Delta \Delta C t}$ & Control & Ethanol \\
\hline p38MAPK & $1.02 \pm 0.07(9)$ & $1.02 \pm 0.10(9)$ \\
SAPK/JNK & $1.03 \pm 0.09(9)$ & $1.07 \pm 0.11(9)$ \\
ERK1/2 & $1.02 \pm 0.07(9)$ & $0.95 \pm 0.13(9)$ \\
MMP-9 & $1.16 \pm 0.20(9)$ & $0.99 \pm 0.19(9)$ \\
MMP-2 & $0.92 \pm 0.13(8)$ & $0.91 \pm 0.11(9)$
\end{tabular}

Values are the means \pm S.E.M. Number within parentheses indicates the number of isolated preparations. GAPDH gene was used as housekeeping gene.

Table 2 Effects of SP600125, SB203580 and PD98059 on the $\mathrm{E}_{\max }$ (\% contraction $\mathrm{KCl}$ $120 \mathrm{mM})$ and $\mathrm{pD}_{2}\left(-\log \mathrm{EC}_{50}\right)$ values for $\mathrm{ET}-1$ in the CSM from control and ethanoltreated rats.

\begin{tabular}{|c|c|c|c|c|}
\hline \multirow[b]{2}{*}{ Inhibitor } & \multicolumn{2}{|c|}{ Control } & \multicolumn{2}{|c|}{ Ethanol } \\
\hline & $\mathrm{E}_{\max }$ & $\mathrm{pD}_{2}$ & $\mathrm{E}_{\max }$ & $\mathrm{pD}_{2}$ \\
\hline None & $21.6 \pm 0.5(4)$ & $7.6 \pm 0.3$ & $36.1 \pm 2.7(5)^{\mathrm{a}}$ & $7.2 \pm 0.2$ \\
\hline SP600125 $(100 \mu \mathrm{M})$ & $20.4 \pm 1.7(6)^{b}$ & $7.1 \pm 0.4$ & $25.4 \pm 3.2(5)^{b}$ & $7.7 \pm 0.5$ \\
\hline SB203580 $(100 \mu \mathrm{M})$ & $22.8 \pm 2.2(6)^{b}$ & $6.9 \pm 0.2$ & $36.4 \pm 3.3(6)^{\mathrm{a}, \mathrm{c}}$ & $6.8 \pm 0.2$ \\
\hline PD98059 $(100 \mu \mathrm{M})$ & $24.6 \pm 1.7(5)^{b}$ & $7.6 \pm 0.6$ & $40.1 \pm 2.3(6)^{\mathrm{a}, \mathrm{c}}$ & $6.6 \pm 0.1$ \\
\hline \multirow{2}{*}{\multicolumn{5}{|c|}{ 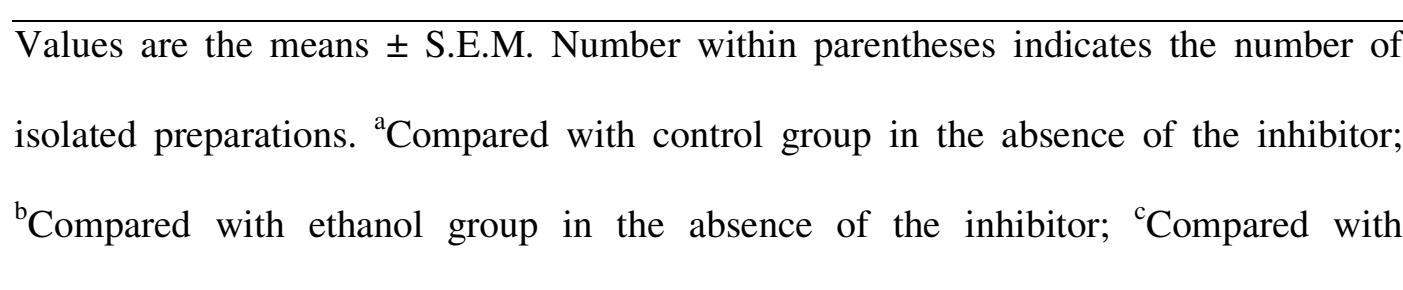 }} \\
\hline & & & & \\
\hline \multicolumn{5}{|c|}{ respective control in the presence of the inhibitor $(\mathrm{p}<0.05$; ANOVA followed by } \\
\hline
\end{tabular}




\section{Legends for figures}

Figure 1. Effects of chronic ethanol consumption on MAPK expression and phosphorylation in the rat CSM. Top panels: representative immunoblots for MAPK protein phosphorylation and expression. Bottom panels: corresponding bar graphs show densitometric data for expression and phosphorylation of p38MAPK (A and B), SAPK/JNK (C and D) and ERK1/2 (E and F). The results are shown as the means \pm S.E.M. of 4 to 8 experiments. ${ }^{*}$ Compared to control ( $\mathrm{p}<0.05$, Student's $t$ test).

\section{Figure 2. Effects of chronic ethanol consumption on MMP and TIMP expression in} the rat CSM. Top panels: representative immunoblots for MMP-9, MMP-2, TIMP-1 and TIMP-2 protein expression. Bottom panels: corresponding bar graphs show densitometric data for expression of MMP-9 (A), MMP-2 (B), TIMP-1 (C) and TIMP-2 (D). Bar graphs E and F represent MMP-9/TIMP-1 ratio and MMP-2/TIMP-2 ratio, respectively. The results are shown as the means \pm S.E.M. of 4 to 9 experiments. *Compared to control ( $\mathrm{p}<0.05$, Student's $t$ test).

Figure 3. Effects of chronic ethanol consumption on gelatinolytic activity and MMP-2 activity in the rat CSM. Representative photomicrographs (captured at $\times 400$ ) of CSM sections incubated with dye-quenched (DQ)-gelatin (A). Quantification of DQgelatin fluorescence in the rat CSM (B). Representative zymogram of MMP-2 in the rat CSM. The marker lane shows the bands corresponding to gelatinases (225, 92 and 72 $\mathrm{kDa}$ ) in the rat CSM. Standard shows the $72 \mathrm{kDa}$ band (MMP-2) and the $92 \mathrm{kDa}$ band (MMP-9), which was used to normalize the data and allow comparisons between gels (C). Corresponding bar graphs show densitometric data for MMP-2 in the rat CSM (D). 
The results are shown as the means \pm S.E.M. of 7 to 10 experiments. ${ }^{*}$ Compared to control ( $\mathrm{p}<0.05$, Student's $t$ test).

Figure 4. Effects of chronic ethanol consumption on plasma MMP and TIMP levels. Plasma levels of MMP-9 (A), MMP-2 (B), TIMP-1 (C) and TIMP-2 (D) were measured by ELISA. Bar graphs E and F represent plasma MMP-9/TIMP-1 ratio and MMP-2/TIMP-2 ratio, respectively. The results are shown as the means \pm S.E.M. of 7 to 10 experiments. *Compared to control ( $\mathrm{p}<0.05$, Student's $t$ test).

Figure 5. Effects of chronic ethanol consumption on MMP-9 (A) and MMP-2 (B) activity in the rat plasma. Right panels: representative zymograms of MMP-9 and MMP-2 in the plasma of rats. The marker lane shows the bands corresponding to gelatinases $(225,92$ and $72 \mathrm{kDa}$ ) in whole blood. Standard shows the $72 \mathrm{kDa}$ band (MMP-2) and the $92 \mathrm{kDa}$ band (MMP-9), which was used to normalize the data and allow comparisons between gels. Left panels: corresponding bar graphs show densitometric data for MMP-9 and MMP-2 in the plasma of rats. The results are shown as the means \pm S.E.M. of 7 to 10 experiments. 
A

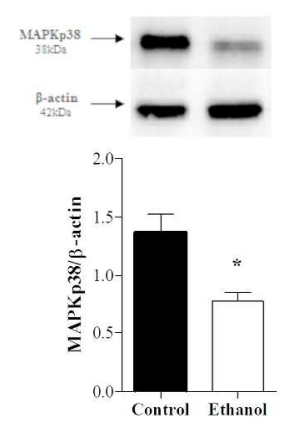

C

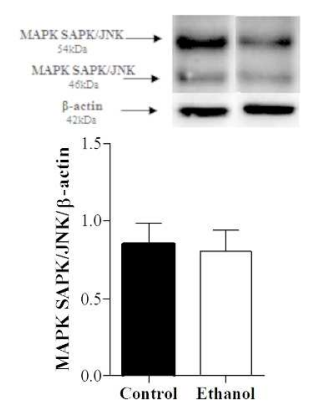

E
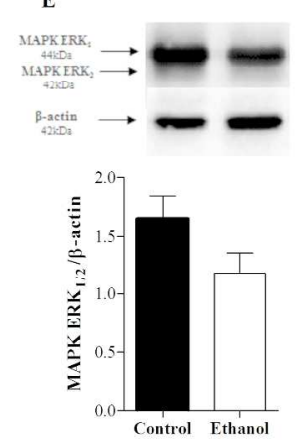

B

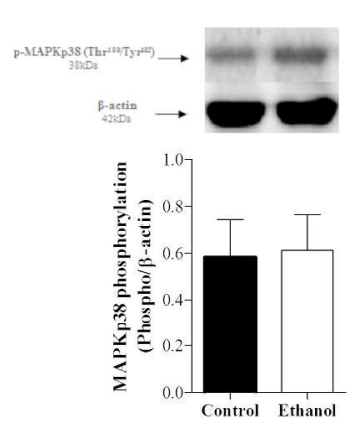

D

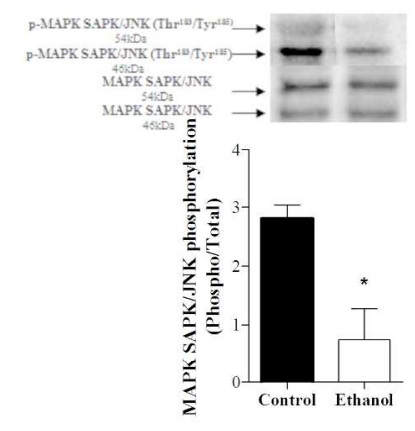

F

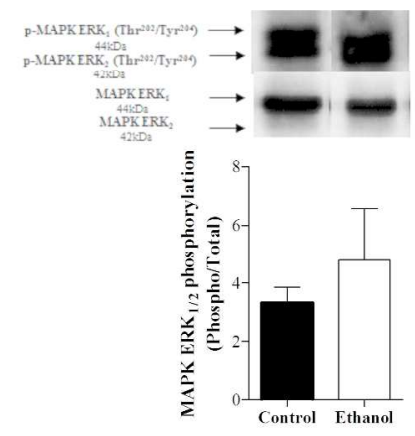

Figure 1. Effects of chronic ethanol consumption on MAPK expression and phosphorylation in the rat CSM. Top panels: representative immunoblots for MAPK protein phosphorylation and expression. Bottom panels: corresponding bar graphs show densitometric data for expression and phosphorylation of p38MAPK (A and $B)$, SAPK/JNK ( $C$ and $D)$ and ERK1/2 (E and F). The results are shown as the means \pm S.E.M. of 4 to 8 experiments. ${ }^{*}$ Compared to control $(p<0.05$, Student's t test).

$158 \times 272 \mathrm{~mm}(300 \times 300$ DPI) 
A

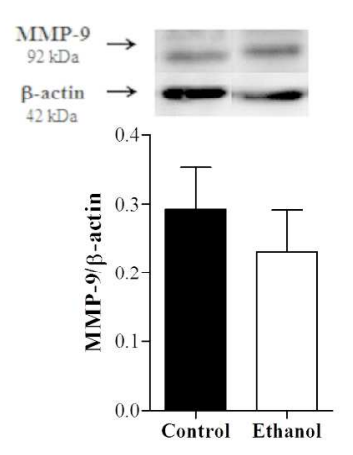

$\mathrm{C}$

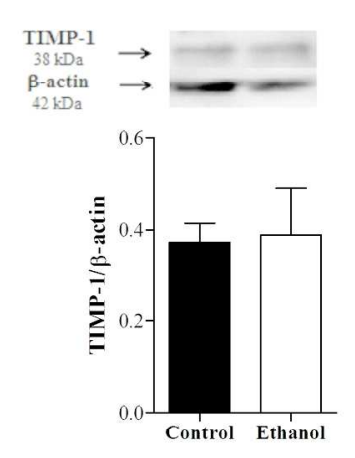

E

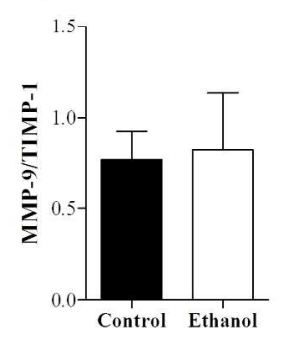

B

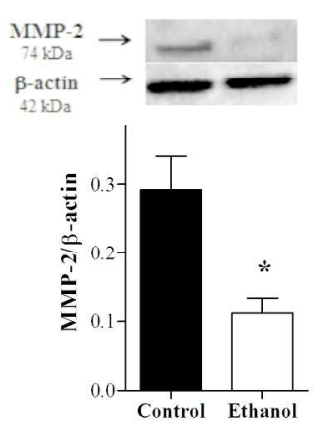

D
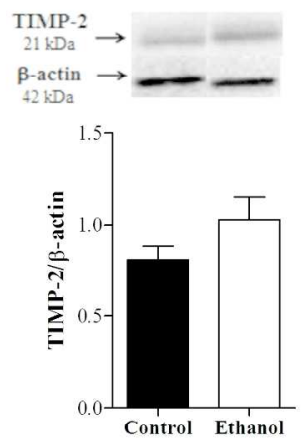

F

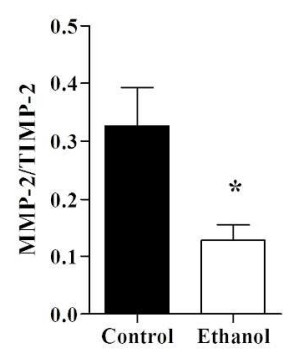

Figure 2. Effects of chronic ethanol consumption on MMP and TIMP expression in the rat CSM. Top panels: representative immunoblots for MMP-9, MMP-2, TIMP-1 and TIMP-2 protein expression. Bottom panels: corresponding bar graphs show densitometric data for expression of MMP-9 (A), MMP-2 (B), TIMP-1 (C) and TIMP-2 (D). Bar graphs $E$ and $F$ represent MMP-9/TIMP-1 ratio and MMP-2/TIMP-2 ratio, respectively. The results are shown as the means \pm S.E.M. of 4 to 9 experiments. ${ }^{*}$ Compared to control $(p<0.05$, Student's $t$ test).

$163 \times 256 \mathrm{~mm}(300 \times 300 \mathrm{DPI})$ 
A

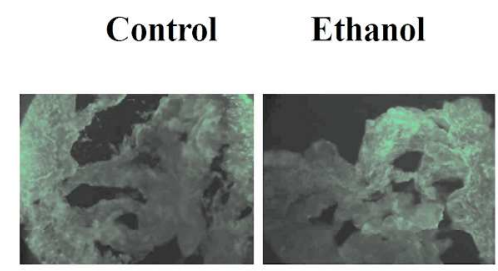

B

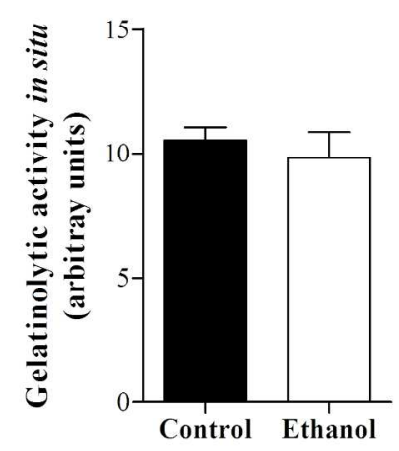

D

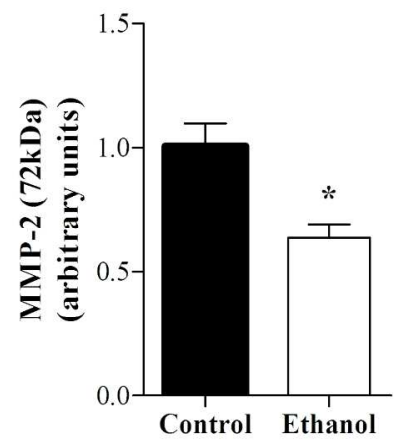

Figure 3. Effects of chronic ethanol consumption on gelatinolytic activity and MMP-2 activity in the rat CSM. Representative photomicrographs (captured at $\times 400$ ) of CSM sections incubated with dye-quenched (DQ)gelatin (A). Quantification of DQ-gelatin fluorescence in the rat CSM (B). Representative zymogram of MMP2 in the rat CSM. The marker lane shows the bands corresponding to gelatinases (225, 92 and $72 \mathrm{kDa})$ in the rat CSM. Standard shows the $72 \mathrm{kDa}$ band (MMP-2) and the $92 \mathrm{kDa}$ band (MMP-9), which was used to normalize the data and allow comparisons between gels (C). Corresponding bar graphs show densitometric data for MMP-2 in the rat CSM (D). The results are shown as the means \pm S.E.M. of 7 to 10 experiments.

*Compared to control ( $p<0.05$, Student's t test).

$$
211 \times 255 \mathrm{~mm}(300 \times 300 \text { DPI) }
$$


A

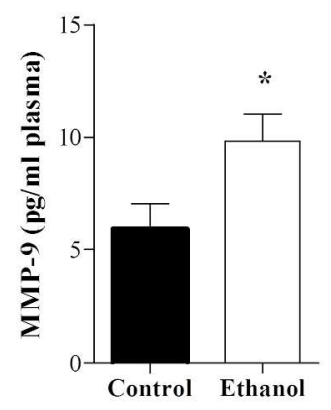

C

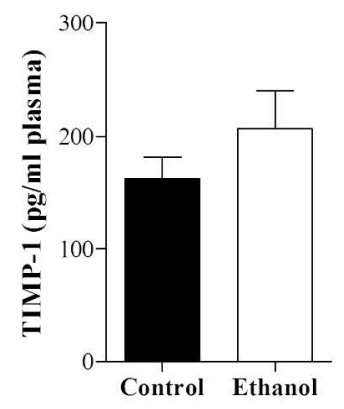

E

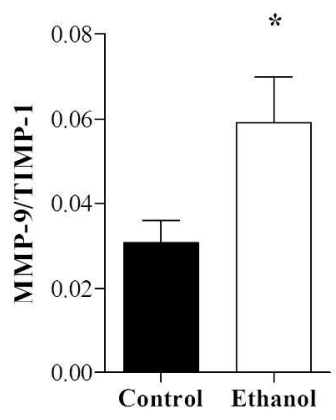

B

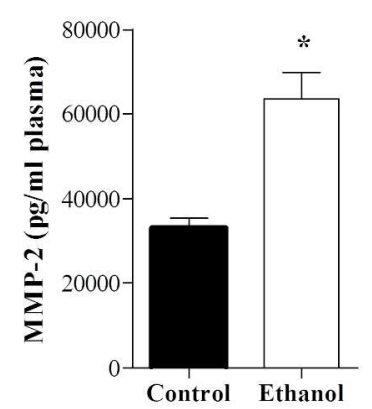

D

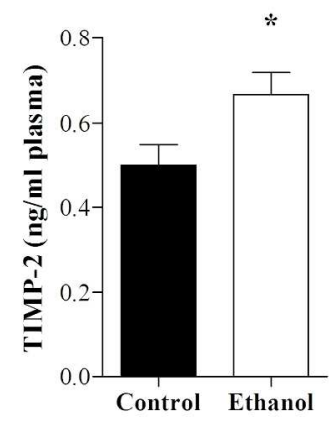

$\mathbf{F}$

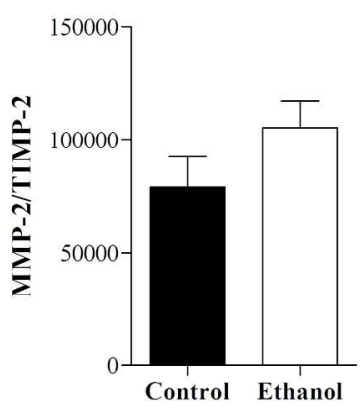

Figure 4. Effects of chronic ethanol consumption on plasma MMP and TIMP levels. Plasma levels of MMP-9 (A), MMP-2 (B), TIMP-1 (C) and TIMP-2 (D) were measured by ELISA. Bar graphs E and F represent plasma MMP-9/TIMP-1 ratio and MMP-2/TIMP-2 ratio, respectively. The results are shown as the means \pm S.E.M. of 7 to 10 experiments. *Compared to control ( $p<0.05$, Student's $t$ test).

$$
186 \times 271 \mathrm{~mm}(300 \times 300 \text { DPI) }
$$


A
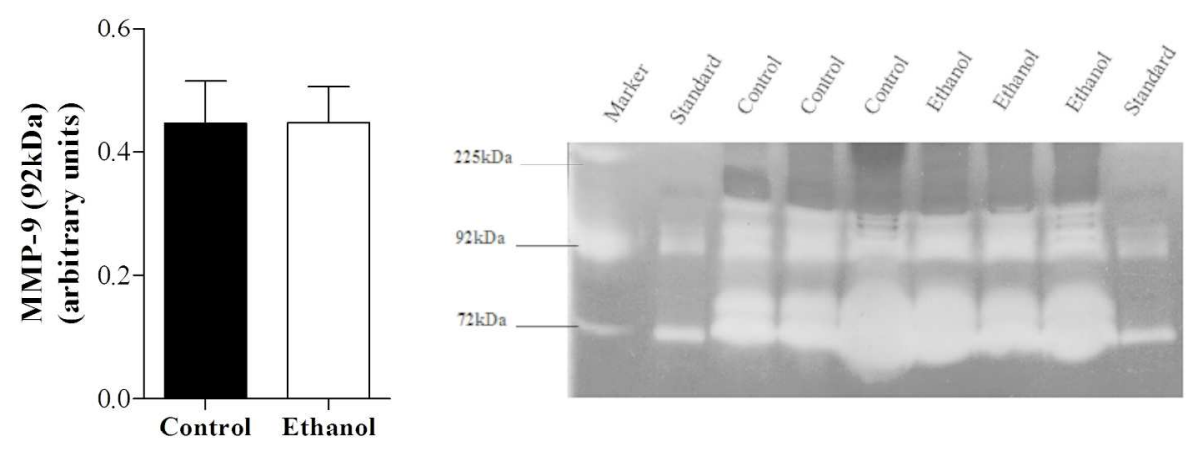

B
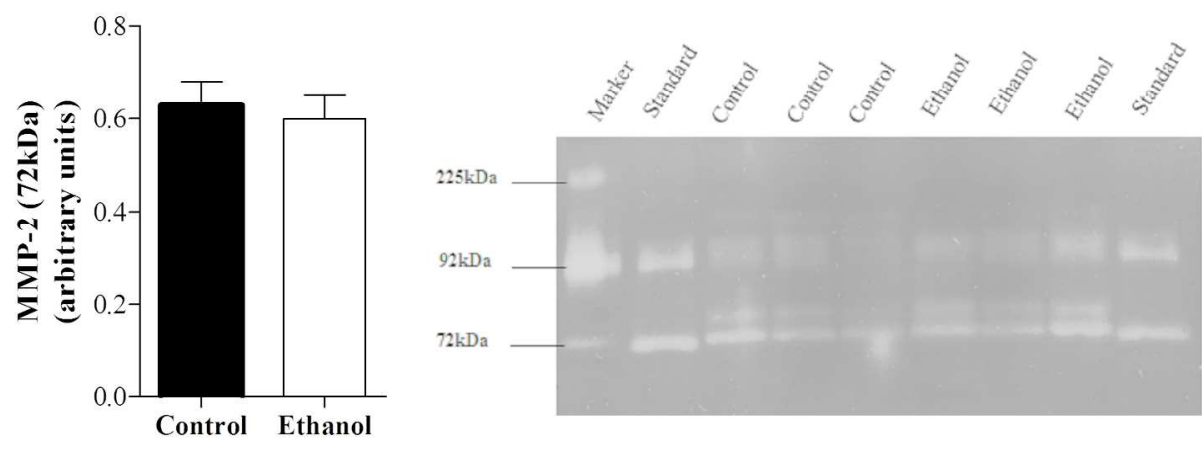

Figure 5. Effects of chronic ethanol consumption on MMP-9 (A) and MMP-2 (B) activity in the rat plasma. Right panels: representative zymograms of MMP-9 and MMP-2 in the plasma of rats. The marker lane shows the bands corresponding to gelatinases (225, 92 and $72 \mathrm{kDa}$ ) in whole blood. Standard shows the $72 \mathrm{kDa}$ band (MMP-2) and the $92 \mathrm{kDa}$ band (MMP-9), which was used to normalize the data and allow comparisons between gels. Left panels: corresponding bar graphs show densitometric data for MMP-9 and MMP-2 in the plasma of rats. The results are shown as the means \pm S.E.M. of 7 to 10 experiments.

$$
201 \times 184 \mathrm{~mm}(300 \times 300 \mathrm{DPI})
$$

